\title{
El "otro": identidad y construcción discursiva
}

\author{
Irene Vasilachis de Gialdino ${ }^{1}$
}

\section{RESUMEN}

Esta contribución, que arraiga en la Epistemología del Sujeto Conocido, tiene por objetivo dar cuenta de las características que asumen textualmente las representaciones discursivas acerca del "otro". De modo tal, luego de la propuesta de tres niveles de reflexión se analizarán, por un lado, las diferentes concepciones que sustentan a esas representaciones y las posibles consecuencias de esas formas de representación, en especial sobre la identidad personal y colectiva y sobre la capacidad de acción histórica de las personas pobres. Por otro lado, se examinará la relación entre las particularidades de dicha representación y las posibles formas de ser de la violencia.

Palabras clave: Epistemología del Sujeto Conocido, identidad, "otro", representaciones sociales, personas pobres, discriminación, violencia.

\begin{abstract}
From the Epistemology of the Known Subject, the author aims at giving account of the traits discourse representations about the "other" assume. After a three-level thinking proposal, different conceptions behind these representations and possible consequences of these ways of representation are discussed, especially from the individual and collective identity on the historic action capability of the poor. Relationship between particulars of said representation and possible displays of violence are also analysed.
\end{abstract}

Key words: Epistemology of the Known Subject, identity, "the other", social representations, the poor, violence.

${ }^{1}$ Ceil-piette (conicet-argentina) 


\section{Revista CUHSO volumen $11 \mathrm{n} \% 1$}

\section{1. - La propuesta de tres niveles de reflexión}

Estimo que el problema de la construcción discursiva del "otro", en especial en los textos científicos puede ser abordado, en principio, mediante la propuesta de tres niveles de reflexión: 1. el ontológico, 2. el epistemológico y 3. el ético-pragmático. Estos niveles de reflexión que se conjugan, son parte de la práctica cotidiana de la investigación y están presentes en el proceso cognitivo ligado a la representación discursiva.

A nivel de la reflexión ontológica, una opción de este carácter es la que subyace a la determinación acerca de, por ejemplo: a) quién es ese "otro" al que se representa; b) cuáles son las características de su identidad; c) en qué difiere o se asemeja de "nosotros"; d) cuáles son sus capacidades de acción y de transformación en el contexto de la sociedad; e) cuál es la "legitimidad" o la "justicia" del lugar que ocupa en ella y de las condiciones en la que desarrolla su existencia.

Cuando el investigador apela a alguno/s de los distintos paradigmas o teorías vigentes en las ciencias sociales es en ellos que encuentra, por lo general, el fundamento de las particularidades identitarias, de las posibilidades sociales e históricas, de ese "otro" al que se refiere. Esto es así en la Epistemología del Sujeto Cognoscente, respecto de la que he predicado la coexistencia de paradigmas (Vasilachis de Gialdino, 1992), y que incluye las formas legitimadas de conocer provistas por esos paradigmas. En el momento actual, lo que postulo es la coexistencia de epistemologías y considero que la Epistemología del Sujeto Cognoscente y la Epistemología del Sujeto Conocido son complementarias y que esta última viene a hablar cuando la Epistemología del Sujeto Cognoscente no tiene palabras para dar cuenta de la identidad, de las percepciones, de los sentidos, de los significados, de las acciones, de las creaciones, de las construcciones, de las representaciones del sujeto conocido.

La Epistemología del Sujeto Conocido produce, pues, una ruptura ontológica a nivel de las características de la identidad del ser humano, al considerar que la identidad posee dos componentes: el esencial y el existencial. Mientras el primero constituye el elemento común que identifica a los hombres/mujeres como hombres/mujeres y los iguala a los otros hombres/mujeres, el segundo constituye el aspecto diferencial que distingue a cada hombre/mujer de los otros hombres/mujeres y lo/ a hace único/a, en su diferencia, frente a todos ellos (Vasilachis de Gialdino, 2003:26).

Es dable afirmar que gran parte de las perspectivas ontológicas acerca de la identidad, vigentes en las ciencias sociales, se centran en su componente existencial; de allí que los resultados de las investigaciones tiendan más a señalar las diferencias entre grupos e individuos que a dar cuenta de aquello que todas las personas tienen en común y que constituye la fuente del respeto a su dignidad.

A nivel de la reflexión epistemológica, necesario es poner de resalto que la forma de acceder al conocimiento de ese "otro" depende, fundamentalmente, de la opción ontológica realizada. Los aspectos que se intente conocer acerca de la identidad de ese "otro" van a depender de las características atribuidas a ésta por las teorías a las que investigador ha recurrido. Las representaciones creadas por esas teorías preanuncian aquello que va a ser encontrado, dibujan el rostro del sujeto conocido antes de que el suj eto cognoscente se aproxime a él y determinan, en gran medida, el comportamiento y las actitudes, las preguntas y las observaciones, las palabras, las pausas y los gestos del sujeto cognoscente.

Por su parte, el método previsto en esas teorías precisa, entre otros: a) cómo y qué se habrá de conocer, b) dónde reside la fuente de conocimiento, c) cómo ese conocimiento se legitima, d) qué constituye una evidencia, e) quiénes han de ser reconocidos como autoridad en el texto científico, f) qué voces han de ser incluidas y g) qué voces han de ser silenciadas. 
La ruptura con la epistemología tradicional que propongo, basada en un diverso planteo ontológico que admite de la presencia conjunta de dos componentes en la identidad, me lleva a extender el principio de la igualdad esencial al proceso de conocimiento y a postularlo respecto de los sujetos de la interacción cognitiva, es decir, la que tiene lugar durante dicho proceso entre el sujeto cognoscente, esto es, el investigador, y el sujeto conocido. En esa interacción dos -o más- personas con igual capacidad de conocer se comunican y mediante esa comunicación amplían y profundizan, conjuntamente, su conocimiento acerca del otro, acerca de la capacidad y de las formas de conocer, acerca del proceso de conocimiento y acerca de sí mismos en lo que ambos sujetos tienen de idéntico. Durante la interacción cognitiva, entonces, personas esencialmente iguales, con disímiles trayectorias existenciales, realizan aportes diferentes.

A nivel de la reflexión ético-pragmática, lo que correspondería preguntarse es para qué y para quién se realiza la investigación, cuál es el propósito de la producción de conocimiento, quién se beneficia con los resultados obtenidos $y$, por tanto, quién es el interlocutor imaginario de los textos científicos -aquél de quien, por lo general, se espera una evaluación positiva-, en fin, quién es el receptor de la representación discursiva del "otro". Esa representación supone tanto ubicar a ese "otro" en relación con el conjunto de la sociedad como asimismo dar por cierta la vigencia de un determinado modelo de sociedad, el que se verá cuestionado, admitido o fortalecido mediante dicha representación.

Es frecuente que la representación del "otro" se efectúe en términos talesque coadyuve a la producción de una imagen positiva de aquél que construye discursivamente a ese "otro", al que, comúnmente, el que elabora el texto, muestra distinto de él mismo a nivel social, educativo, cultural, económico. Es habitual, también, que ese "otro" permanezca ausente como interlocutor imaginario de los textos científicos y que poco se midan las consecuencias que sobre su identidad personal, social, laboral, política, producen las representaciones que se construyen acerca de él.

Este proceso reflexivo, que va de uno a otro de los tres niveles, es hábil para impedir, por ejemplo, que la opción ontológica que el investigador realiza, la que se vincula con la identidad que reconoce a ese "otro", sea tergiversada por la presión epistemológica ejercida sobre la construcción de ese "otro" en el texto científico, el que es, por lo común, el reflejo discursivo de una ontología realista. Ese texto suele responder a los modos de conocimiento propiosdel paradigma positivista, los que tienden más a la "objetivación" que a la "personificación", que a la "subjetivación", del sujeto conocido. Dado el predominio de la perspectiva de dicho paradigma en las ciencias sociales, esos modos de conocimiento difícilmente son abandonados cuando se asumen otras orientaciones epistemológicas.

\section{2.- Las formas que asume la representación del "otro"}

En el supuesto de que la opción ontológica del investigador sea la propuesta por la Epistemología del Sujeto Conocido, ese investigador reconocerá los componentes esencial y existencial propios de la identidad $y$, por consiguiente, la igualdad esencial de los sujetos que participan en el proceso de conocimiento. Se hallará, entonces, ante la necesidad de revisar aquellos criterios de validez asociados con la observación de las diferencias entre los individuos, para establecer, más tarde, regularidades a partir de esas diferencias y terminar, muchas veces, consagrando, cuando no justificando, esas diferencias en desmedro de lo que asemeja a todos los hombres y mujeres y los identifica como seres humanos.

Gran parte de las investigaciones e informes sobre la pobreza, por ejemplo, dan cuenta de los rasgos que comparten las personas pobres y que las distinguen de otras, sin señalar el carácter existencial, contingente, de esas diferencias. Por lo además, se 
señalan respecto de esas personas pobres, en especial, características negativas de distinta índole, marcadas argumentativamente mediante el paradigma de la negación. Lo que, generalmente, no se indica, con pareja intensidad, es la presencia de características positivas entre las que se hallan las que les permiten a las personas pobres enfrentar cotidianamente la situación de privación en la que se encuentran y, al mismo tiempo, luchar denodadamente para que sus hijos no tengan que atravesarla en el futuro.

En consonancia con las propuestas de la Epistemología del Sujeto Cognoscente, los que se indican, frecuentemente, respecto de las personas pobres, son los que denomino procesos "des" que suponen, en gran medida, a la sociedad como un sistema. De esta suerte, adquieren sentido oposiciones tales como socialización/ desocialización; estructuración/ desestructuración; integración/ desintegración, entre otras (Vasilachis de Gialdino, 2003:95-96; 2006:484-485).

Cuando esos procesos, consecuencia de las relaciones de privación a las que se ven sometidas las personas pobres en un contexto social determinado espacial y temporalmente, se analizan de acuerdo con la Epistemología del Sujeto Conocido, desde la perspectiva de quienes los padecen, se advierte, contemporáneamente, el surgimiento de otro tipo de procesos a los que designo como procesos "re", esto es, de resistencia, de reivindicación, de redescubrimiento de habilidades, de restablecimiento de vínculos sociales, de redefinición de la propia identidad frente a las representaciones creadas en torno a ellos. Esta práctica se puso de manifiesto como resultado de realizar el análisis lingüístico de las entrevistas que constituían el corpus de la investigación sobre "Extrema pobreza en la ciudad de Buenos Aires". La resistencia discursiva desplegada por los actores, Ios llevaba, por un lado, a definir su identidad por oposición, esto es, a determinar qué aspectos, qué características que se les atribuían "no" formaban parte de su identidad $y$, por el otro, a diferenciar, por medio del uso de distintos verbos, el "ser de la calle" del "estar en la calle", es decir, a distinguir los aspectos esenciales de los existenciales de la identidad.

Entiendo que si la mención de los procesos "des" no se efectúa acompañada de la de los procesos "re" se limita, se recorta, se tergiversa la identidad de las personas pobres, se las discrimina, a través de una acción que también es de privación de identidad, y que viene a fortalecer el entramado de las relaciones de ese mismo carácter al que las personas pobres están sometidas.

\section{3.- Ética y representación}

La decisión del investigador acerca de si representa al "otro" señalando las diferencias que lo separan de él y de otros y/o mostrando aquello en lo que él y ese "otro" se asemejan y se identifican, tiene un fundamento ontológico, pero, principalmente, significativas consecuencias éticas.

Mientras el reconocimiento de la igualdad funda la consagración de la paridad de derechos, por el contrario, la marcada indicación de la diferencia puede servir a intereses opuestos y conducir a que los derechos de unos se asienten sobre aquellos que a otros les son negados. Los procesos discriminatorios se caracterizan por intentar, primero, mostrar las diferencias que separan a unos de otros grupos, a unos de otros individuos para justificar, después, la necesidad de reprimir, de controlar, de someter, de aislar, de excluir, de negar, a aquellos que no se consideran como iguales, a aquellos que, con su sola presencia, constituyen una amenaza para los bienes y los valores en los que sustentan su posición quienes discriminan.

No sólo la violencia reconocida como tal en sus distintas manifestaciones sino, también, el olvido y la indiferencia, como otras formas de ser de la violencia, apartan a esos "otros", a los que se ubica en la "periferia", de un "nosotros" a quienes se reserva el lugar del "centro". Lo más probable es que en ese 
UNIVERSIDAD CATÓLICA DE TEMUCO

"centro" también se sitúe el investigador, a pesar de recorrer una y otra vez la "periferia" durante el trabajo de campo, haciéndose cargo de la relevancia ética de constituirse, las más de las veces, en un mediador cuya sola presencia, cuya acción, cuya omisión, cuya práctica discursiva, cuya actividad académica, puede llevar a unir, a ligar o, contrariamente, a distanciar, a aislar a esos mundos considerados como separados el uno del otro. Justamente, es durante el trabajo que he observado cómo las situaciones de pobreza surgen por todos los intersticios de la sociedad, que no se exhibe un "adentro" separado de un "afuera" y que la propuesta de una definición relacional de esas situaciones permite ubicar a las personas pobres, a sus relaciones, a sus acciones en el corazón mismo de la sociedad, en su núcleo vital, en el lugar en que se toman las decisiones, consagrándose, de esta manera, su posibilidad, su capacidad, en fin, su derecho de participar en ellas.

Quienes optan por subrayar, desde la perspectiva del sujeto conocido, lo que iguala, lo que identifica a unos con otros seres humanos, concluyen en que la mayor parte de las diferencias existenciales que impiden el desarrollo autónomo de la identidad de las personas pobres son la consecuencia de las acciones de privación de otros respecto de ellas (Vasilachis de Gialdino, 2003).

Las formas de representar a los "otros" se sustentan, por lo habitual, en las dos concepciones a las que he aludido. Una marca, en particular, la diferencia, y la otra se basa en el respeto al principio de igualdad. Ambas concepciones suponen distintos modelos de sociedad, promueven valores e identidades diferentes. La primera relega la identidad esencial y destaca las diferencias existenciales, la segunda, pone en la identidad esencial el fundamento de la existencia humana. Esta última perspectiva impide que se sancionen derechos, potestades, prerrogativas a la medida de la protección de los bienes de diversa índole de quienes justifican en la diferencia a su favor el mantenimiento de la desigualdad. Por este medio, dicha orientación intenta vedar la salvaguardia de una injusticia que predomina allí donde la igualdad esencial es negada, confinada, omitida.

El discurso es una práctica social y el investigador también puede llevar a cabo acciones injustas -al desconocer el principio de igualdad- como resultado de su indiferencia respecto de las consecuencias que sus palabras podrían provocar sobre la identidad del "otro", sobre sus posibilidades de acción, de participación, de decisión, de definición tanto de las que considera sus necesidades como del modo de satisfacerlas ¿Cómo podrían las personas pobres reclamar el respeto a su dignidad si se reitera, una y otra vez, que están sometidas a procesos ineludibles cuyo sentido no pueden modificar, a situaciones que no están en condiciones de transformar a través de su propia acción? ¿No estará, también, el investigador, mediante las representaciones que construye, consagrando diferencias aparentemente insalvables que lo separan de ese "otro" y que lo ubican en un lugar de privilegio respecto de su propia situación y en cuanto a su capacidad de comprensión de la situación, de las perspectivas, de las expectativas del "otro"?

La apelación a diversas metáforas -geométricas, náuticas, físicas, biológicaspara categorizar, calificar, conceptualizar a las personas pobres determina que se borren las diferencias individuales entre esas personas y que, al unísono, se intensifiquen las diferencias que las separan respecto del conjunto de la sociedad y de quien emplea esas metáforas. Estimo que, en estos supuestos, aún sin proponérselo, el investigador puede, a través de su práctica discursiva, estar realizando una acción de privación de identidad, esto es, violando el principio de igualdad esencial a través del recurso de mostrar como esenciales las diferencias que predica textualmente, respecto de "otros", como existenciales (Vasilachis de Gialdino, 1999a:58, 2003:101).

Desde la Epistemología del Sujeto Conocido se cuestiona toda relación que suponga superioridad de unos sobre otros 
individuos y que, por lo tanto, desconozca el principio de igualdad. En este sentido, se cuestionan las interacciones cognitivas que: a) desconozcan esa igualdad; b) presupongan el mayor valor de una forma de conocer sobre otras y c) asignen al que emplee esas formas de conocer, consideradas como más valiosas, un poder legitimado como para disponer del conocimiento del que lo provee el sujeto conocido, independientemente de los efectos que pueda producir sobre la identidad, situación y relaciones de éste.

El investigador debe, pues, interrogarse acerca de si es posible representar discursivamente a ese "otro", en particular cuando ese "otro" es una persona pobre, sin, al mismo tiempo, mostrar la injusticia de toda distribución que permita a unos vivir a costa, o con indiferencia, del ataque a la dignidad de otros.

Las categorías, estereotipos, nociones, creadas por diferentes órdenes de discursos -científico, mediático, político, filosófico, jurídico, entre otros- median, se interponen, como toda representación, entre el sujeto cognoscente y el sujeto conocido durante el proceso de conocimiento. Impiden, de esta suerte, la libre manifestación de ese sujeto conocido al mismo tiempo que la conmoción del sujeto cognoscente. Cuando esas representaciones dan prioridad al componente existencial de la identidad sobre el esencial tienden a fortalecer, a consolidar, cuando no a justificar, la desigualdad. En lo que hace a su función social no sólo sirven para legitimar el lugar que ocupan quienes las producen sino que, además, cuando son internalizadas por quienes acceden a esos discursos, transforman el conocimiento de éstos, empañan su mirada, cierran sus manos y producen el olvido, la negación, el rechazo de aquellos "otros" que las tienen abiertas hacia ellos esperando el gesto de aceptación, de comprensión, de entrega, esto es, de reconocimiento como iguales del que son privados.

\section{4.- Violencia y representación}

Distintas investigaciones que he llevado a cabo sobre las representaciones creadas por la prensa escrita de la República Argentina respecto, en especial, de los trabajadores (Vasilachis de Gialdino, 1997, 1999b, 1999c, 2001, 2002), de las personas pobres (Vasilachis de Gialdino, 1999a, 1999b, 2001, 2003); de los niños que trabajan y/o viven en la calle (Vasilachis de Gialdino, 2003) y de los actores que manifiestan, reclaman, reivindican en los conflictos sociales (Vasilachis de Gialdino, 2005); sumadas a aquellas realizadas para determinar las características que en la prensa escrita de El Salvador adquiere la construcción de representaciones sociales acerca de la identidad de los jóvenes a los que se vincula con actos delictivos (Vasilachis de Gialdino, 2004), me han llevado a concebir, a partir de los datos de las mencionadas investigaciones, un conj unto de aportes teórico-epistemológicos encaminados a comprender el problema de la violencia. Las conclusiones comunes a todas estas investigaciones fueron las siguientes: a) el predominio de las acciones de privación de identidad en la construcción discursiva de la identidad de los sujetos cuya representación se analizaba; b) la tendencia a producir una imagen negativa de ellos, señalando lo que los diferencia en desmedro de lo que los identifica con el resto de los individuos; c) la ampliación y consolidación de las estrategias y de los procesos discriminatorios; d) la negación a unos y el reconocimiento a otros del derecho de participar como iguales y libres en los procesos de construcción de la sociedad y, en fin, e) el ejercicio de la violencia a través del reiterado desconocimiento del principio de igualdad esencial.

Esa violencia no sólo está presente en lo que se dice y en cómo se lo dice sino, también, en lo que se oculta, en lo que se tergiversa, en lo que se niega, en aquello que se construye como real más allá de toda realidad.

En consonancia con la Epistemología del Suj eto Conocido, la discriminación es también una acción violenta. A través de ella, uno de los sujetos de la relación social desconoce el componente esencial, común, idéntico de la identidad del otro sujeto o niega, rechaza, no tolera, su diferencia existencial. 
UNIVERSIDAD CATÓLICA DE TEMUCO

El discurso discriminatorio tiene como característica la construcción y/ o reproducción de un núcleo cognitivo adecuado para justificar el rechazo, la separación, la negación, el aislamiento del "otro" mediante el recurso de mostrar las características que lo diferencian de "nosotros".

Cuanto menos representaciones
alternativas, cuanto menos modelos
interpretativos que intenten cambiar el
horizonte de significado respecto de las
características de determinadas personas
o grupos estén vigentes en el mundo de la
vida como esquemas comprensivos de su
situación, de sus relaciones, de sus acciones,
en fin, respecto de su identidad esencial
y existencial, más enraizados estarán los
procesos discriminatorios. La peculiaridad
de estos procesos es, precisamente, proveer
de recursos cognitivos mostrados como
incuestionables e insubstituibles a los que se
debe acudir para referirse a esas personas o
grupos discriminados y para orientar la acción
dirigida a ellos.

Esos recursos cognitivos tienen, por lo general, la particularidad de generalizar, respecto del grupo discriminado, características negativas de las que el grupo que discrimina carece. Estos atributos justifican tanto el lugar que este úl timo grupo ocupa en la sociedad como su situación de privilegio en lo que concierne a la distribución y a la asignación de los bienes valorados socialmente. El empleo de esos recursos cognitivos tiene, entonces, respecto de la sociedad en su conjunto, la función de reproducir valores, jerarquizaciones, formas de poder, de control y de dominación.

Respecto de quien emplea esos recursos cognitivos con efectos discriminatorios se da un doble movimiento: por un lado, de afirmación de su propia identidad existencial y de su posición social y, por el otro, de negación de la identidad esencial de los "otros" - al no reconocerlos como iguales- $y$, por ende, de impugnación de sus derechos, de sus atributos y de sus posibilidades de existencia (Vasilachis de Gialdino, 2003:177).
En los textos analizados sociológica y lingüísticamente, la discriminación se lleva a cabo por medio de la utilización de distintos recursos como, por ejemplo: a) la forma en la que ese "otro" es calificado, categorizado, definido; b) las metáforas a las que se recurre para nombrarlo; c) los roles pasivos que se le adjudican; d) las acciones, en su mayoría, contrarias a las normas y expectativas sociales que se le atribuyen $y / 0$ que se predican de él y e) los procesos y situaciones a los que se los muestra como sometidos de manera ineludible.

El empleo de estos recursos da cuenta, a nivel textual, de las opciones ontológicas, epistemológicas y ético-pragmáticas del hablante y, portanto, del modelo interpretativo de la realidad social que subyace en su discurso. Esos modelos suponen: a) alguna forma de ser de la sociedad y de la organización social, b) uno o varios modos de diferenciación o de jerarquización entre sus miembros, c) un tipo de relaciones sociales predominante $y$, por tanto, d) una mayor o menor posibilidad de los actores sociales individuales o colectivos, por un lado, de contribuir con la construcción de la sociedad, de sus valores, de sus normas, de sus significados, de sus orientaciones $y$, por el otro, de proponer y obtener una transformación en los sistemas de distribución de bienes tanto materiales cuanto simbólicos, espirituales y de trascendencia (Vasilachis de Gialdino, 1997:301).

De tal manera, las representaciones acerca de la sociedad, de sus relaciones, de la legitimidad de éstas, de las identidades individuales y colectivas, de la mayor o menor posibilidad de los individuos de desarrollarse autónomamente, entre otras, son construidas textualmente a partir de la adhesión a los presupuestos de dichos modelos 0 , más bien, a los paradigmas y teorías en los que esos modelos se sustentan.

Así, por ejemplo, la representación discursiva, generalmente en términos negativos, de los niños que trabajan y/o viven en la calle, en la prensa escrita de la Argentina 
aparece ligada a una premisa subyacente, por un lado, como recurso cognitivo empleado por el hablante $y$, por el otro, como recurso al que debe apelar el lector del texto para comprender el sentido de las emisiones contenidas en ese texto. Dicha premisa, que no comparto, puede ser formulada de la siguiente manera: cuanto mayor es la exclusión de un grupo social menor será la posibilidad de que sus miembros modifiquen sus comportamientos "desviados". Como se puede advertir, ese recurso cognitivo es hábil para consolidar procesos discriminatorios, los que tienen la particularidad de incorporar al mundo de la vida un conjunto de modelos interpretativos que se asientan sobre variados presupuestos acerca de la acción de las personas y/o grupos discriminados pero, además, sobre el valor de verdad acordado a una suma de relaciones causales -meramente hipotéticas pero mostradas como probadas y ciertasreferidas a la vinculación entre esas acciones y una serie de determinantes familiares, sociales, contextuales, históricos, biológicos, genéticos, raciales, de género, entre otros, que, ineludiblemente, producen esas acciones (Vasilachis de Gialdino, 2003:190).

Es habitual que en los textos científicos se empleen al unísono, para representar a las personas pobres, varios de los recursos a los que he aludido y que poseen efectos discriminatorios. Pero, además, es necesario poner de resalto, que muchos de esos recursos están ya presentes en las teorías a las que el investigador recurre, de allí la necesidad de los tres niveles de reflexión que propongo.

Cuando con el uso de esos recursos se ataca, fundamentalmente, al componente común, esencial, de la identidad se produce una acción violenta. Esta violencia que no es, generalmente, considerada como tal promueve toda suerte de injusticia. Por medio de ella se somete a unos respecto de otros a estigmatizaciones, a categorizaciones, a definiciones, a conceptualizaciones, a estereotipos que no pueden cuestionar. El que esa acción no sea reputada como violenta impide, por una parte, que el que la comete reconozca y evite su ejercicio y, por la otra, que los que la sufren reivindiquen su derecho a ser protegidos, defendidos respecto de ella.

\section{CONCLUSIONES}

Un examen de las estrategias discursivas utilizadas para describir al "otro" se impone, pues, también, en el mundo de la ciencia. Los procesos discriminatorios se traducen en mecanismos de control social a través de los cuales se elabora y se consolida la "identidad" como identidad que al individuo le es adjudicada, que se interpone entre él y el resto de la sociedad en las relaciones sociales que entabla y que, por tanto, él no construye existencial y libremente. Esa identidad que le es impuesta se caracteriza por reunir los requisitos que aseguran el mantenimiento de un modelo de sociedad y de organización social, que asegura la protección de los intereses de aquellos que han tenido y tienen en su manos la posibilidad de fij ar los términos de la asignación de los diversos tipos de bienes y de recursos.

Las formas, los procesos, los métodos de conocimiento suponen violencia sobre otros cuando mediante su empleo se desconoce la igual capacidad de conocer de todos los individuos $y$, por tanto, se niega a algunos la aptitud reflexiva que les permite tomar decisiones acordes con la total dimensión de su identidad, con el completo despliegue de sus componentes esencial y existencial. Sería, pues, una acción violenta, entre otras, la que tergiverse la identidad, la situación, las necesidades, las posibilidades, las perspectivas, las propuestas, el sentido de la acción, el conocimiento aportado por el sujeto conocido.

Es dable sostener que la preponderante tendencia del conocimiento científico a mostrar las semejanzas en las diferencias, siendo ésta una condición de las generalizaciones, y a los sujetos como objetos, siendo ésta una condición de la objetividad, lo ha llevado a descuidar el señalamiento del aspecto esencial de la identidad. Por este medio 
UNIVERSIDAD CATÓLICA DE TEMUCO

ha sido frecuente la esencialización de las diferencias existenciales entre los individuos, la que se ha traducido, por una parte, en la imposición, sobre muchos, del peso de la ignorancia, de la culpa, de la irracionalidad, de la indolencia, de la incapacidad y, por la otra, en el otorgamiento, a pocos, del derecho a decidir, a sancionar, a resolver, a juzgar, a disponer tanto de su propio destino como del de los demás.

Considero que los que intentan conocer a la sociedad, a sus situaciones, a sus procesos, a sus actores, a la identidad de éstos, deberían interrogarse acerca del valor, acerca de las consecuencias, acerca de las funciones de los presupuestos sobre el carácter necesario de determinadas relaciones sociales, sean éstas de solidaridad, de cohesión, de integración, de negociación, de cooperación, de oposición, de conflicto, de antagonismo. La sociedad, sus formas posibles de ser, habrían de concebirse, más bien, como una construcción colectiva en la que todos, como iguales y libres, pueden contribuir en la búsqueda y en la obtención del bien común. Múltiples luchas recientes, variadas acciones de resistencia, innumerables conflictos han tenido, precisamente, como finalidad el reconocimiento de esa igualdad, la aspiración a que las acciones de unos no nieguen lo que el "otro" es, puede, espera, necesita, reclama.

Considero que en la construcción discursiva del "otro" los investigadores han de poner de manifiesto, a la vez, a ambos componentes de la identidad del sujeto conocido, a la presencia de los procesos "des", conjuntamente con la de los procesos "re", al carácter cooperativo de la construcción del conocimiento en las ciencias sociales, al elemento común que iguala al investigador con ese "otro" y que los identifica, a ambos, como seres humanos. Si esa manifestación no se plasma, es posible que sobre la coherente, organizada, apretada trama de los enunciados del texto científico se modele y se erija un mundo ajustado sólo a las necesidades de los que asientan sus derechos sobre la negativa de los derechos de otros.

\section{BIBLIOGRAFIA}

VASILACHIS DE GIALDINO, I. (1997). La construcción de representaciones sociales: el discurso político y la prensa escrita. Barcelona: Gedisa.

VASILACHIS DE GIALDINO, I. (1999a) "Las acciones de privación de identidad en la representación social de los pobres. Un análisis sociológico y lingüístico". Discurso y Sociedad, 1(1): 55-104.

VASILACHIS DE GIALDINO, I. (1999b) "La construcción de identidades en la prensa escrita. Las representaciones sociales sobre los trabajadores y los pobres o las otras formas de ser de la violencia". Sociedad 15: 65-101.

VASILACHIS DE GIALDINO, I. (1999c) "La construcción de representaciones sociales sobre el mundo del trabajo: discurso político y prensa escrita". Tareas 101: 35-60.

VASILACHIS DE GIALDINO, I. (2001) "Pobres, trabajo y representaciones sociales". En Forni, F. y Angélico, H. (comps) Articulaciones en el mercado laboral. Buenos Aires: CEIL-La Colmena.

VASILACHIS DE GIALDINO, I. (2002) "Trabajo e identidad: reflexiones epistemológicas a partir de la investigación empírica". Sociología del Trabajo 44: 3-39.

VASILACHIS DE GIALDINO, I. (2003). Pobres, pobreza, identidad y representaciones sociales. Barcelona: Gedisa.

VASILACHIS DE GIALDINO, I. (2004) “EI lenguaje de la violencia en los medios de comunicación: las otras formas de ser de la violencia y la prensa escrita". En Aportes para la convivencia y la seguridad ciudadana. San Salvador: PNUD(ONU)-Programa Sociedad sin violencia-PRODECA.

VASILACHIS DE GIALDINO, I. (2005). La representación discursiva de los conflictos sociales en la prensa escrita. Estudios Sociológicos, XXIII(67): 95-136.

VASILACHIS DE GIALDINO, I. (2006) “Identity, poverty situations and the Epistemology of the Known Subject". Sociology, 40 (3): 473-491. 


\section{NOTA BIOGRÁFICA}

Irene Vasilachis de Gialdino es Doctora en Derecho, Socióloga y especialista en análisis del discurso, es docente de Postgrado de distintas Universidades de su país y del exterior. Es Investigadora Principal del Consejo Nacional de Investigaciones Científicas y Técnicas (CONICET) de la Argentina, en el Centro de Estudios e Investigaciones Laborales (CEIL-PIETTE). Sus trabajos están realizados desde una perspectiva interdisciplinaria en la que se conjugan el derecho, la sociología y la lingüística. Sus áreas de interés son la epistemología, las metodología cualitativa, el análisis lingüístico del discurso, la creación mediática y política de representaciones sociales, la pobreza y los conflictos sociales. Entre sus principales publicaciones ligadas a la temática de esta contribución se encuentran: Métodos Cualitativos. Los problemas teórico-epistemológicos (1992), Buenos Aires, CEAL; La construcción de representaciones sociales: el discurso político y prensa escrita (1997), Barcelona, Gedisa; Pobres, pobreza, identidad y representaciones sociales (2003), Barcelona, Gedisa; La representación discursiva de los conflictos sociales en la prensa escrita (2005), Estudios Sociológicos XXIII (67), 95-136; Identity, poverty situations and the Epistemology of the Known Subject (2006), Sociology, 40 (3), 473-491 y Estrategias de investigación cualitativa (coord.) (2006), Barcelona, Gedisa. 